In Crescendo. Institucional. 2016; 3(1): 71-76

Fecha de recepción: 6 de mayo de 2016

Fecha de aceptación: 17 de mayo de 2016

\title{
Caracterización y EVOlución de la teCnOlogía edUCATIVA EN EL PERÚ
}

\section{Characteristics and EVOlution of educational TECHNOlOGy IN PERU}

\author{
Mario Enrique Nizama Reyes"
}

\begin{abstract}
RESUMEN
T a presente investigación tuvo como objetivo determinar las principales características Lde la tecnología educativa y describir la evolución que esta ha presentado en nuestro país. El diseño de la investigación fue no experimental, descriptivo, bibliográfico y documental; por lo tanto, no hay población, tampoco muestra. La caracterización de la tecnología educativa se realizó a través del detalle de cinco postulados, dos enfoques y dos fundamentos. Respecto a la evolución de la tecnología educativa, esta abarcó dos periodos específicos, de los años 70 a los 90 y de los 90 a la actualidad. En cuanto a las conclusiones se puede indicar en primer lugar que los objetivos deben ser diseñados para promover cambios en el estudiante. En segundo lugar, que los enfoque están centrados en los medios y en la instrucción. También se pudo concluir que la tecnología educativa posee fundamentos filosóficos y psicológicos. Para concluir, indicar que en nuestro país la tecnología educativa aún no se ha logrado consolidar y menos aún aplicarse de manera adecuada en la mayor parte de nuestro sistema educativo.
\end{abstract}

Palabras clave: tecnología educativa, enseñanza, aprendizaje.

\begin{abstract}
This research aimed to determine the main characteristics of educational technology and describe the evolution that is presented in our country. The research design was not experimental, descriptive, bibliographical and documentary; therefore, no population, not shown. The characterization of educational technology was performed using five postulates detail two approaches and two foundations. Regarding the evolution of educational

* Docente tutor de la Universidad Católica Los Ángeles de Chimbote, filial Piura. menizamar@gmail.com
\end{abstract}


technology, this included two specific periods, of the 70 to 90 and 90 to the present. As for the findings it may indicate at the outset that the objectives should be designed to promote changes in the student. Second, that approach are focused on the media and instruction. It might also conclude that educational technology has philosophical and psychological underpinnings. In conclusion, in our country indicate that educational technology has not yet been consolidated, let alone implemented appropriately in most of our education system.

Keywords: educational technology, teaching, learning.

\section{INTRODUCCIÓN}

La tecnología educativa es una disciplina que integra la teoría y la práctica del diseño y desarrollo, selección y utilización, evaluación y gestión de los recursos tecnológicos aplicados al proceso de enseñanza-aprendizaje, cuyo fin es el de contribuir a la mejora de las actividades educativas y la resolución de sus problemas (Fernández, 2006).

Ante el imperante, imponente y globalizado uso actual de la tecnología en casi todos los procesos humanos y obviamente los procesos sociales, un proceso tan primordial y trascendente como el proceso educativo (enseñanza-aprendizaje) no puede quedar exento de la incorporación tecnológica con la finalidad de mejorar su eficiencia y eficacia, y obviamente aumentar el rendimiento y desempeño tanto del docente como del estudiante.

Pero la realidad nos indica que en nuestro sistema educativo nacional, el empleo adecuado y eficiente de la tecnología educativa aún es incipiente o está mal orientada, pues esta debe variar de acuerdo con las necesidades sociales y debe ser una respuesta a estas necesidades educativas. Si bien es cierto, desde los años noventa han habido esfuerzos reales por implementar una adecuada tecnología educativa, lamentablemente no ha existido una política definida, constante y continua que permita mejorar los resultados de nuestros estudiantes.

En la actualidad una de las mejores alternativas de tecnología educativa a implementar en nuestro país son las LMS (Learning Management System) o sistema de gestión de aprendizaje, que son conjuntos de módulos de software, que se ejecutan sobre un servidor web (internet) con la finalidad de administrar, ejecutar y controlar diversas actividades educativas de manera continua, fomentando así la interacción asíncrona y la retroalimentación entre el docente y el estudiante y también entre los propios estudiantes participantes, lo que enriquece este proceso de comunicación. Las LMS más representativas son Moodle y Dokeos.

También cabe indicar que no basta con implementar la mejor tecnología educativa, sino que el factor humano es la piedra angular del éxito; así pues el docente aparte de ser un profesional capaz de seleccionar, operar y evaluar tecnologías, debe estar capacitado y concientizado en potenciar las buenas relaciones humanas, pues no siempre las dificultades del aprendizaje se relacionan con los objetos del conocimiento, sino también por efecto de una baja edificación de afectividad primaria entre los agentes educativos. Cuando se aplica una tecnología educativa humanizante los resultados finales del proceso educativo son más positivos, se aprende más y mejor. Cuando las relaciones entre profesor 
y estudiante son meramente racionales, sin implicancia afectiva, el aprendizaje es menor en cantidad y calidad, especialmente en educación inicial y primaria, pero no se excluye la secundaria y tampoco la superior (Calero, 2009).

Por todo lo antes expuesto y detallado, se justifica el presente trabajo de investigación, puesto que consolidar e implantar una tecnología educativa acorde a nuestras realidades y necesidades educativas se hace imperiosa con la finalidad de lograr eficiencia y eficacia en los procesos educativos, planteándose el siguiente problema: ¿Cuáles son los elementos y componentes que caracterizan la tecnología educativa en nuestra realidad y como ha sido su evolución en nuestro país? Para dar respuesta a este problema, se planteó el siguiente objetivo general: Determinar las principales características de la tecnología educativa y describir la evolución que esta ha presentado en nuestro país.

\section{MATERIAL Y MÉTODOS}

El diseño de la investigación fue no experimental, descriptivo, bibliográfico y documental; por lo tanto, no hay población, tampoco muestra. Asimismo, el enfoque de la investigación fue cualitativo y el nivel descriptivo.

En el desarrollo de la presente investigación se han utilizado los textos y manuales de diversos autores referidos a tendencias y paradigmas educativos, así como de la tecnología educativa en nuestro país.

\section{RESULTADOS}

Iniciando el desarrollo de la caracterización de la tecnología educativa en nuestro país en la Tabla 1 se esquematizan los cinco postulados que la rigen.

Tabla 1. Postulados de la tecnología educativa

\begin{tabular}{|c|c|}
\hline N. ${ }^{\circ}$ & Ítem \\
\hline 1 & $\begin{array}{l}\text { Los objetivos deben ser diseñados para promover cambios en el estudiante, en atención a su } \\
\text { personalidad integral y fines del sistema educativo. }\end{array}$ \\
\hline 2 & $\begin{array}{l}\text { La tecnología promueve que el estudiante alcance el dominio de la materia con apoyo del docente. } \\
\text { El éxito del estudiante es el éxito del docente. }\end{array}$ \\
\hline 3 & $\begin{array}{l}\text { Los estudiantes aprenden en distintas formas y velocidades y cada tipo de instrucción tiene sus } \\
\text { propias características de aprendizaje. }\end{array}$ \\
\hline 4 & $\begin{array}{l}\text { Los elementos del proceso educativo deben ser vistos en forma integral. Todo lo que le sucede al } \\
\text { estudiante en su centro de estudios, hogar o comunidad debe poder relacionarse en su educación. }\end{array}$ \\
\hline 5 & $\begin{array}{l}\text { Cualquier sistema de aprendizaje debe tener incorporado dispositivos de evaluación y comunicación } \\
\text { de retorno que suministren información permanente para mejorar el sistema. }\end{array}$ \\
\hline
\end{tabular}
Fuente: Peñaloza, 1991

Asimismo y continuando con la caracterización de la tecnología educativa, en la Tabla 2 se desarrollan sus dos enfoques. 
Tabla 2. Enfoques de la tecnología educativa

\begin{tabular}{|c|c|c|}
\hline N. ${ }^{\circ}$ & Enfoque & Detalle \\
\hline 1 & $\begin{array}{l}\text { Tecnología educativa } \\
\text { centrada en los medios }\end{array}$ & $\begin{array}{l}\text { Relacionada con el diseño, desarrollo e implementación de } \\
\text { técnicas y materiales (productos) basados en las tecnologías } \\
\text { de información y comunicación para promover la eficacia y la } \\
\text { eficiencia del proceso de enseñanza aprendizaje y contribuir a } \\
\text { resolver los problemas educativos. }\end{array}$ \\
\hline 2 & $\begin{array}{l}\text { Tecnología educativa } \\
\text { centrada en la } \\
\text { instrucción }\end{array}$ & $\begin{array}{l}\text { Relacionada con la planificación y gestión del proceso de } \\
\text { enseñanza aprendizaje aplicando los principios científicos: teorías } \\
\text { de aprendizaje, diseño del currículo, selección y producción } \\
\text { de materiales, elección de métodos, gestión de la instrucción y } \\
\text { evaluación de los resultados. }\end{array}$ \\
\hline
\end{tabular}

Fuente: Peñaloza, 1991.

Como tercera y última parte en la caracterización de la tecnología educativa, en la Tabla 3 se desarrollan los respectivos fundamentos.

Tabla 3. Fundamentos de la tecnología educativa

\begin{tabular}{ccl}
\hline N. & Fundamentos & \multicolumn{1}{c}{ Detalle } \\
\hline 1 & Filosóficos & $\begin{array}{l}\text { La tecnología educativa se fundamenta en el pragmatismo. Todo } \\
\text { el contenido o valor de nuestras ideas o conceptos se reduce a las } \\
\text { consecuencias prácticas que podemos esperar de ellos. } \\
\text { Se fundamenta en el cognitivismo y el constructivismo. Destaca } \\
\end{array}$ \\
& Psicológicos & $\begin{array}{l}\text { la enseñanza programada, que se orienta a que cada estudiante } \\
\text { trabaje según su propio ritmo y posibilidades. }\end{array}$ \\
\hline
\end{tabular}

Fuente: Rivera, 1990.

Respecto a la evolución de la tecnología educativa en nuestro país, esta se desarrolla cronológicamente la Tabla 4.

Tabla 4. Evolución de la tecnología educativa en el Perú

\begin{tabular}{cl}
\hline Periodo & \multicolumn{1}{c}{ Detalle } \\
\hline $\begin{array}{c}\text { De los años } \\
70\end{array}$ & $\begin{array}{l}\text { La tecnología educativa estaba centrada en la instrucción (planificación y gestión } \\
\text { del proceso de enseñanza aprendizaje). Destacan en este periodo Walter Peñaloza, } \\
\text { a los }\end{array}$ \\
Juan Rivera entre otros maestros. \\
\\
De los años & - Programa EDURED (Redes Educativas). \\
90 & - Programa Piloto de Educación a Distancia (EDIST). \\
a la & - Proyecto Huascarán. \\
actualidad & - Dirección General de Tecnologías Educativas (DIGETTE). \\
& - Programa una laptop por niño. \\
\hline
\end{tabular}

Fuente: Calero, 2009 


\section{DISCUSIÓN}

Respecto a los postulados podemos resaltar el referido a que los objetivos de la tecnología educativa deben promover cambios en los estudiantes en atención a su personalidad integral, es decir, se debe buscar un desarrollo completo, que se potencie la mayor cantidad de capacidades, destrezas y habilidades en nuestros estudiante, y no solo una o determinada capacidad. Por ejemplo, por años se tenía la concepción que el estudiante de buen desempeño en matemáticas era "más inteligente", por tanto los currículos y contenidos se orientaban a "formar" estudiantes en matemáticas, descuidando o relegando otras áreas como la educación cívica, la historia o la educación física. ¿Qué se obtuvo como resultado? No pocos ciudadanos "muy inteligentes" pero con mínimos o nulos valores o civismo.

En cuanto a los enfoques, claramente se definen los dos aspectos en los que actualmente la tecnología educativa tiene alcance, los medios y la instrucción. En el actual siglo XXI, y teniendo ya generaciones de estudiantes "nativos digitales" se hace cada vez más prioritario e impostergable la inclusión y sobre todo el dominio por parte del docente, de productos acordes a las nuevas formas de enseñanza, aprendizaje y comunicación como son los software educativos y las diversas herramientas de la web 2.0, herramientas que se caracterizan por ser interactivas y en tiempo real como las ya famosas omnipresentes redes sociales (Facebook, Twitter, etc.), además de los blogs, foros, wikis, y otras, que correctamente implementadas, utilizadas y retroalimentadas promoverán justamente la tan ansiada eficacia y la eficiencia del proceso de enseñanza-aprendizaje. Se debe insistir en que los docentes deben estar lo suficientemente capacitados y preparados para este reto, pues el uso de las TIC y la internet es "jugar en el campo de los estudiantes", por lo cual si no hay un adecuado manejo, todas las herramientas antes mencionadas se volverán en contra y fomentarán efectos negativos como el plagio y mínimo esfuerzo en los estudiantes.

Referente a los fundamentos de la tecnología educativa, se observa que a nivel filosófico se fundamente en el pragmatismo, y ello hace entender el porqué del éxito de las redes sociales entre los jóvenes, pues las consecuencias prácticas que esperan de esas redes son altamente catalogadas por los usuarios, en especial porque resuelve problemas precisos, como la distancia geográfica, y que además permiten una interacción a la vez en tiempo real, bajo una interfaz que genera "protección", la cual es inexistente en una interacción directa. Y en cuanto al fundamento psicológico, las herramientas TIC actuales, específicamente las ya mencionadas redes sociales y, sobre todo, las plataformas de enseñanza de aprendizaje virtual (sistemas de gestión de aprendizaje o LMS por sus siglas en inglés) se basan y fundamentan en el constructivismo, primando la interacción y generación de productos (tareas) a partir de información publicada (libros, revistas y separatas digitales), además de una adecuada exploración de saberes previos y saberes base (mediante blogs y foros). Si a esto le sumamos una adecuada retroalimentación en línea (obviamente con un adecuado dominio por parte del docente no solo del tema sino 
también de las TIC) se puede asegurar que mediante plataformas y aulas virtuales los estudiantes puedan trabajar según su propio ritmo y posibilidades, dando como resultado eficiencia y eficacia en el proceso de enseñanza-aprendizaje.

Finalmente, para la evolución de la tecnología educativa en nuestro país, podemos decir que si bien data desde los años setenta, con grandes maestros que discutían lo positivo y negativo de la tecnología educativa, la realidad es que todavía no ha logrado consolidarse y menos aún aplicarse de manera adecuada en la mayor parte de nuestro sistema educativo. Si bien es cierto que hay excepciones, donde se ha demostrado la viabilidad y eficiencia (sobre todo en entidades privadas), en la gran mayoría de casos los resultados son opuestos, primordialmente por la poca existencia de verdaderos especialistas en tecnología educativa (con comprobada experiencia en nuestra realidad) además de la pobre capacitación realizada y con poco nivel de exigencia y primordialmente el poco compromiso de docentes y estudiantes.

Tomando como base los resultados que se han podido presentar, sistematizar y discutir previamente, como conclusiones del presente trabajo podemos mencionar las siguientes:

- Los postulados de la tecnología educativa son cinco destacando que los objetivos deben ser diseñados para promover cambios en el estudiante y que el éxito del estudiante es el éxito del docente.

- Existen dos enfoques de la tecnología educativa, el centrado en los medios (productos) y el centrado en la instrucción (planificación y gestión). Ambos son complementarios para lograr resultados positivos en el proceso de enseñanzaaprendizaje.

- La tecnología educativa tiene un fundamento filosófico, concretamente el pragmatismo y un fundamento psicológico, específicamente el cognitivismo y el constructivismo.

- En nuestro país la tecnología educativa aún no se ha logrado consolidar y menos aún aplicarse de manera adecuada en la mayor parte de nuestro sistema educativo, lo que la constituye en todo un reto y un problema interesante para la investigación.

\section{REFERENCIAS BIBLIOGRÁFICAS}

Calero, M. (2009). Tecnología Educativa: realidades y perspectivas. Lima, Perú: Editorial San Marcos.

Fernández, B. (2006). Tecnología Educativa: ¿Sólo recursos técnicos? La Habana, Cuba: Editorial ISPEJV.

Peñaloza, W. (1991). Tecnología Educativa. Lima, Perú: Editorial EEACAB.

Rivera, J. (1990). Educación y Tecnología Educativa. Lima, Perú: Editorial ATEI. 